\title{
Systematic Development and Evaluation of a User-Oriented System for Public Transport Vehicles Identification
}

\author{
Alexander Mueller ${ }^{1(凶)}$, Adrian Kemper ${ }^{2}$, Ingrid Bubb ${ }^{3}$, Nour $\mathrm{Sakr}^{3}$, Gerhard Kopp ${ }^{4}$, \\ and Robert Hahn ${ }^{4}$ \\ ${ }^{1}$ Hochschule Esslingen - University of Applied Sciences, Esslingen, Germany \\ Alexander.muellerahs-esslingen.de \\ 2 in $2 \mathrm{p} \mathrm{GmbH}$, Fellbach, Germany \\ ${ }^{3}$ Lehrstuhl für Ergonomie, Technische Universität München, Munich, Germany \\ ${ }^{4}$ Institut für Fahrzeugkonzepte, Deutsches Zentrum für Luft- und Raumfahrt e.V., Stuttgart,
}

Germany

\begin{abstract}
This paper describes how a user-oriented system for identification of public transport vehicles can be developed and evaluated. For this purpose, a vehicle design is first scaled with respect to the project requirements using a proven method and with decided consideration of customer and accessibility requirements. In a next step, it is demonstrated why the recognition of the bus by public transport users at bus stops has to be identified as critical. The following section systematically generates concepts for bus identification and presents them virtually. Finally, it is described how the concept evaluation can succeed with a pedestrian simulator and which results are obtained: For example, a number should do the primary bus identification, the route should be displayed at the boarding and the individual stop destination should be clearly highlighted on the route.
\end{abstract}

Keywords: User modelling · Information exchange · Display and control design - Demand responsive public transport - Autonomously operated public transport · Vehicle identification · User-centered vehicle design

\section{Introduction}

\subsection{Status Quo and Problem Statement}

The high share of individual passenger transport by car leads to traffic jams and high exhaust emissions, especially in urban areas. In order to improve this situation, there is a high level of interest in innovative, individual, environmentally friendly and thus attractive transport systems in society and subsequently among transport operators. One promising approach is to organize public transport according to demand. Advances in the fields of digital networking, autonomous driving and e-mobility are helping to make demand-responsive public transport attractive from a business perspective as well. At present, these systems are supplementing established regular transport services in the form of pilot projects [1] (see Fig. 1). 

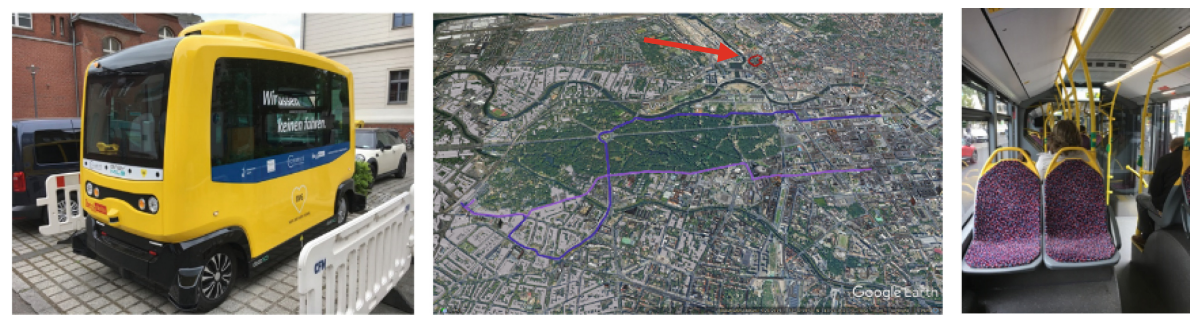

Fig. 1. Examples of progressive and proven public transport applications in Berlin from left to right: 1 . Highly automated vehicle (red route), 2. Driving routes Berlin Center, 3. Interior of a current city bus (blue/purple route)

However, since autonomous public transportation operating models eliminate the bus driver as a companion and contact person, the interaction between the user and the public transportation system must be clear and free of misunderstandings.

\subsection{Objective}

On the one hand, this leads to the question, which interactions have to be considered as critical. In addition, questions are raised regarding the development of possible interface concepts that enable the design-critical interactions with minimal errors, as well as how users evaluate them.

These questions were investigated within the research project RAMONA (Realisierung Automatisierter Mobilitätskonzepte im Oeffentlichen Nahverkehr = Realization of Automated Mobility Concepts in Public Transportation). Through the application of development methods, layout critical design solutions were generated and partially evaluated using virtual reality. This publication especially focuses on the design process and evaluation of the display and operating concept. In particular, it focuses on the interaction between the user and the public transport system at stops and stations.

\subsection{Input Parameter Vehicle Design Methodology}

As the input variable, a generic development approach for generating a vehicle concept for demand-responsive and autonomous public transport, which was defined in the context of the research project Reallabor Schorndorf [2], was used [3]. This method is based on user-centered modeling of the dimensional layout on the basis of main requirements. As a result, the design-critical user scenario is first mapped, taking into account the technical functional assemblies, and then the vehicle geometry is systematically designed inside out by the application of the proven product development method described in VDI 2221 [4].

Due to the changed boundary conditions of the present research project, a variation of the requirements took place. This variation essentially leads to the presentation of a dimensional layout and in consequence, to a vehicle concept with smaller capacity, i.e. 10 seats and standing places including the provision for a wheelchair transport. 
In comparison to the previous work, the requirements definition was extended by integrating the Quality Function Deployment (QFD) approach. In first order, requirements and wishes were derived from the user and operator perspective. For example, the desire for vehicles that can be deployed flexibly and in a demand-driven manner and that are consequently small and maneuverable contrasts with the largest possible capacity, which enables operation with few empty runs.

\section{Identification of Critical Interactions}

\subsection{Information Flow Analysis of a Typical Public Transport Usage}

For the analysis of the information flow, the fundamental schema of human-machine interaction is used as a basis [5]. The sequence and the number of information flows in a public transport use can be variant. For an effective analysis, premises (e.g.: demanddriven \& autonomous operation, ordering via smartphone, etc.) were defined to narrow down the numerous variants in a meaningful way. As an approach, a typical public transport usage was divided over time into sub-processes and their individual interaction loops. Dividing into the sub-processes 1. "Ordering process", 2. "Finding the departure point", 3. "Waiting at the bus stop", 4. "Identification \& preparation for entry", 5. "Boarding", 6. "Ride" and 7. "Exit" has proven to be successful. For these individual subprocesses, the multisensory information exchange was depicted and analyzed. Figure 2 shows an extract of the "Identification" and "Preparation for entry" subprocesses.

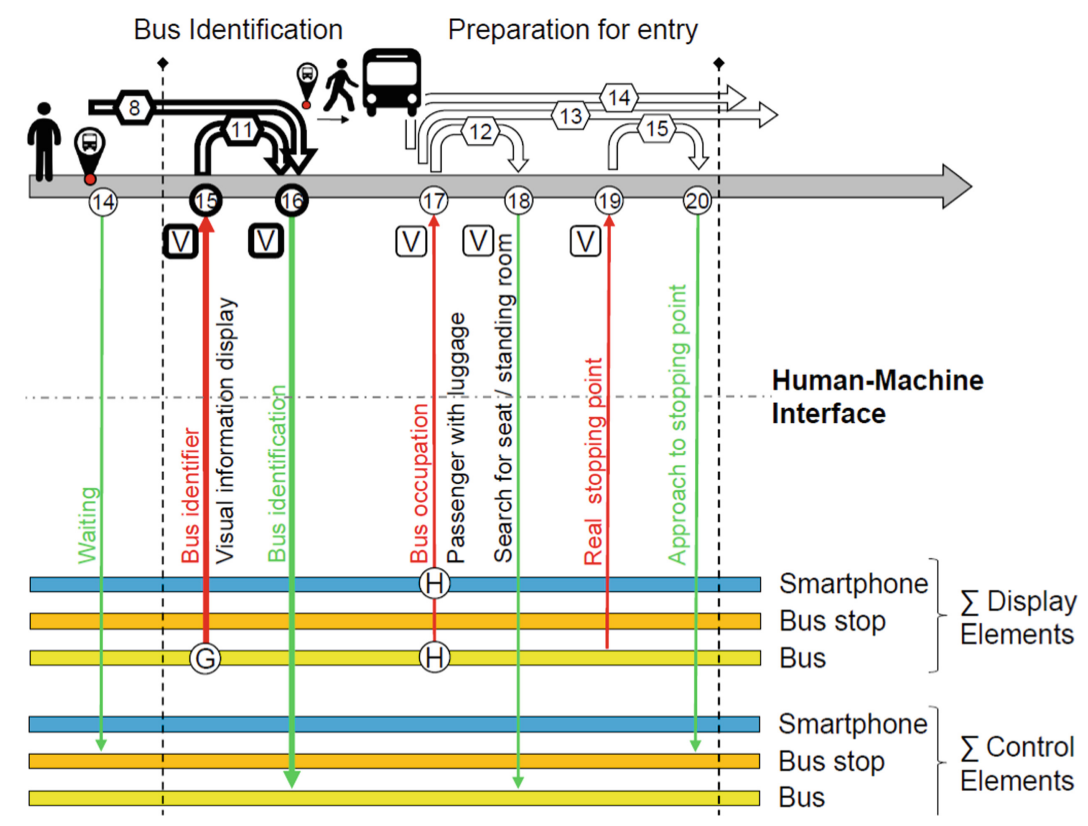

Fig. 2. Information flow diagram of the sub processes "Identification \& preparation for entry". 
In this figure, the perception and behavior of the user is shown at the top, and the display and user interface of the public transport system is shown at the bottom. Interactions across the human-machine interface are distinguished by the colors red and green, depending on the direction. The individual interactions are numbered with circles, where the identification of the perception processes is done by hexagons and the display contents are visualized with letters in circles. Information that is processed during an interaction can originate from directly preceding interaction loops or already from preceding subprocesses, which is represented by arrows above the time axis. As an example, in the "Identification (16)" interaction loop, the bus identifier information from the booking process (8) and the display content of the arriving bus (10) are compared with each other.

Based on these diagrams, the evaluation of the complexity of individual interactions can be determined. The following three hypotheses serve as evaluation criteria: Experience values with the interaction at hand, runtime/duration of the interaction loop, and number of parallel interaction loops. Since these three criteria only apply to the subprocess "Identification \& boarding preparation" for the typical public transportation use described, this sub-process is considered particularly design-critical [6].

\subsection{Observational Study}

Additionally, to the methodical analysis, the authors conducted an observation study to identify interaction difficulties between conventional busses and users. The focus of the study was on minibuses transporting up to 10 people, as this best fitted the objectives and conditions of the project RAMONA. One observer recorded 121 bus stops and the behavior of 432 users at two different bus stops with the help of a self-created observation catalog. Regarding the descriptively analyzed data, the authors defined five main categories of critical passenger patterns: concentration of the passenger during the waiting time, waiting location, behavior reaching the bus, behavior during boarding and exit and necessary communication to the bus. In corresponds to the information flow process the authors could identify the location of departure and the identification of the bus as particularly critical interaction points especially if the bus will be used as an on-demand bus [6].

\subsection{Discussion}

The considerations on the information flow analysis are based in particular on analytical and theoretical background. Also, in the case of the observational study, a limiting factor is that the derived problems are an extrapolation to the challenges that on-demand autonomous buses may face. Nevertheless, it appears to the authors through both theoretical and exploratory analyses that users are concerned with questions such as "Is this really my bus?" or "Where is my bus located?" when it comes to public transportation. Such questions can justify why one of the routine tasks of a bus driver is to communicate with and assist passengers [7]. Therefore, it is necessary to address those needs as well in the context of autonomous vehicles and to conceptualize helpful solutions for users. 


\section{Conception of Control and Display System}

According to the critical interaction point "identification of the bus" identified in Chapter 2, concepts for the information representation were systematically created. The concept creation also followed the procedure published in VDI 2221 [4] and was combined with a morphological product development method [8]. An essential approach was to make the relevant identification contents available to the users in different ways at the right time and partly also in parallel. Essentially, concepts for information encoding and presentation were developed using different display systems (visual displays at the front, side and inside the vehicle as well as mobile phone displays).
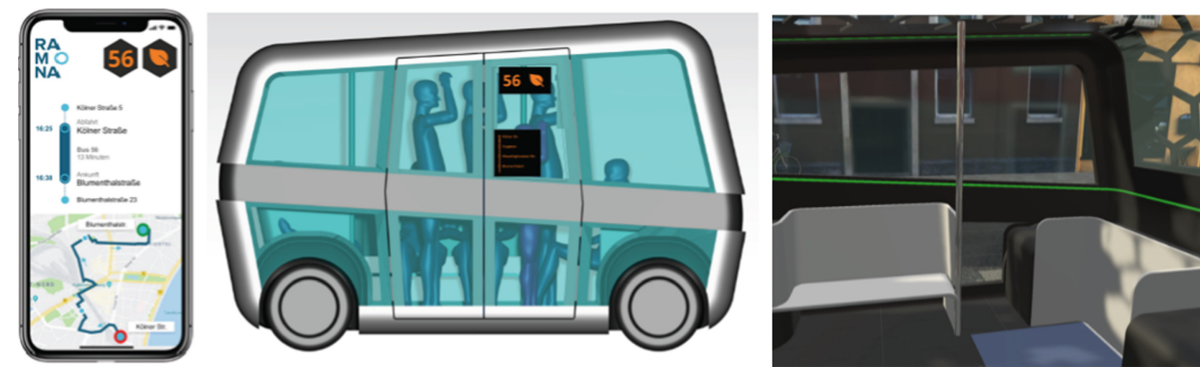

Fig. 3. Chosen information sources from left to right: 1. Mobile phone display (APP), 2. External Bus Interface (eHMI), 3. Interior lighting as feedback (e.g. green light correct bus) (iHMI).

Figure 3 shows the different chosen information levels. The first source of information is a mobile phone display including all necessary information, such as the unique identifier of the bus, route information as well as departure time and location and destination - hereafter referred to as $A P P$. The second source is the unique bus identification on the bus (depending on the concept with more or less content) - henceforth called $e H M I$. The last source is a feedback system to confirm the correct entry of the passenger - hereinafter $i H M I$. If a passenger enters the correct bus, a green light flashes for confirmation in the interior of the bus; if they enter the wrong bus, a red light appears.

\section{Concept Evaluation in Pedestrian Simulator}

The authors evaluated the developed concepts by means of a virtual reality (VR) study from the perspective of a bus passenger (i.e. a pedestrian simulator). Subjects experienced a bus arrival and boarding scenario via a head-mounted display (HTC Vive Pro Eye). In the $V R$ world, test persons could make use of an $A P P$ with all information concerning their travel whenever they needed, different $e H M I s$ on the front and side of the bus with different degrees of content and an $i H M I$ feedback system inside the bus (see Fig. 3). The $V R$ simulation was programmed in Unity and the experimental scenario placed participants at a virtual bus stop in Munich city center. To investigate the research questions the authors chose a between-subjects design with different degrees of content for the $e H M I$. Table 1 summarizes the various interface designs as well as the utilized measures. Each subject experienced only one of the three $e H M I$ identifiers. 
Table 1. Collected data of study.

\begin{tabular}{l|l}
\hline$e H M I / H H M I$ & Measures \\
\hline $\begin{array}{l}\text { 1. Number on front and side of the bus } \\
\begin{array}{l}\text { 2. Number and symbol on front and side of } \\
\text { the bus }\end{array}\end{array}$ & $\begin{array}{l}\text { Efficiency - based on how often the virtual } \\
\text { smartphone } A P P \text { needed to be prompted for } \\
\text { each concept }\end{array}$ \\
$\begin{array}{l}\text { Effectiveness - based on test subjects' entry } \\
\text { into the correct or wrong bus } \\
\text { the bus with an additional schematic route } \\
\text { showing the next stops on the side of the } \\
\text { bus }\end{array}$ & $\begin{array}{l}\text { Preferred concept - Test subjects from each } \\
\text { group could indicate which of the three } \\
\text { concepts types they prefer after the experiment }\end{array}$ \\
\hline $\begin{array}{l}\text { 4. Light in the interior for bording } \\
\text { confirmation }\end{array}$ & $\begin{array}{l}\text { iHMI - Helpfulness of the feedback system } \\
\text { (Five-Point Likert Scale) }\end{array}$ \\
\hline & $\begin{array}{l}\text { Qualitative feedback and design } \\
\text { recommendations }\end{array}$ \\
\hline
\end{tabular}

\subsection{Experimental Setup}

After an initial training phase, test subjects received a written instruction, that they had ordered a bus to a specific address. The booking confirmation (APP) was presented to the subjects, so they could use it to identify the bus in the VR scenario. Subsequently, participants were asked to play a puzzle game on a mobile phone to allow for distraction. Five minutes later, they entered the $V R$ and were told to board the bus that led to their destination. In the $V R$ there was already a bus waiting at the stop. It had a different identifier from the one on the APP. Subjects could then decide whether to enter this bus or wait for the right one. The latter arrived one minute later. Using a remote control, subjects could call up the $A P P$ with information about the identifier of their bus. As soon as a subject boarded a bus, they received visual feedback on whether they were on the right or wrong bus via the $i H M I$. Figure 4 illustrates the starting condition of a subject for the Concept 3 group.

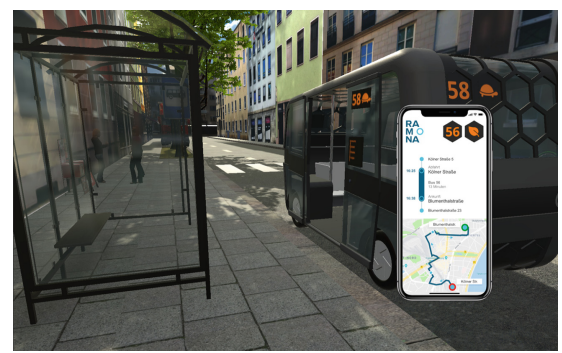

Fig. 4. Wrong automated bus waiting at the bus stop with an $e H M I$. 


\subsection{Results}

Sixty subjects ( $46.67 \%$ women) participated on the experiment. They were manually divided into three groups of 20 persons each. There were $65 \%$ students in each group. The age of the test persons ranged between 20 and 60 years $(M=27.4$ years, $M d N=25)$. $80 \%$ of the subjects already experienced some form of on-demand mobility (e.g. Uber). Based on the measures described in Table 1, the following results could be reported:

Efficiency - 30\% of all subjects boarded the wrong bus. However, logistic regression shows no significance between the concepts $((\beta=.24 ; \mathrm{p}=.49$; Wald $=0.688)$.

Effectiveness - The number of $A P P$ views per user group was examined. A Kruskal-Wallis test identifies no significance (Chi-Square $(2)=3.42, \mathrm{p}=.181$ ).

Preferred concept - Across all groups, the third concept could be identified as the preferred concept (75\%). A Kruskal Wallis test confirms this with significance (Chi-Square $(2)=7.60, \mathrm{p}=.022)$. The second $e H M I$ concept was only chosen by Group 2 , i.e. only those who had experienced it.

$i H M I$-the majority of participants (78\%), who had made an error when boarding the bus, strongly agreed that the $i H M I$ lighting was helpful. No subject, who entered the wrong bus, disagreed that the $i H M I$ was helpful. Of those who only experienced the green light (correct bus), only $10 \%$ disagreed that the lighting was helpful (7\% strongly disagreed, $2 \%$ disagreed).

\section{Qualitative feedback and design recommendations.}

1. $e H M I$ - the subjects prefer an interface including a number and route. As the bus is on-demand, the passenger's individual stop destination should be clearly highlighted on the route display.

2. $i H M I$ - additionally to the responsive lightning (correct bus - green vs. Wrong bus - red) a route display should be visible inside the vehicle, including all the stops of all passengers.

3. APP - participants would like a signal on their smartphone to let them know when their bus is arriving. They also requested to be able to track the movement of the bus on their smartphones.

\subsection{Discussion}

In order to be useful, a product should fulfil the pillars of efficiency, effectiveness, and satisfaction [9]. Even though the $e H M I$ concepts did not have statistically significant differences in terms of efficiency and effectiveness, the results showed significant differences in terms of satisfaction across the groups (preferred concept). Nonetheless, according to the subjective feedback the proposed concepts, especially Concept 3 , seem to fulfil their purpose successfully.

Applying a between-subjects design was necessary to avoid learning effects. Evidently, all participants recognized the correct bus after making an error with the first one. That is why due to the nature of the experiment, repeated measures were not possible. This also led to a reduced number of data points and test persons per experimental group $(n=20)$, which does not allow any large generalizations to be made. 


\section{Conclusion}

As a result of the research activities outlined, the following conclusions emerge:

1. The existing development methodology for the systematic generation of the vehicle concept was successfully applied on the basis of specified main requirements.

2. Using two independent methods to detect critical interaction behaviors between users and the public transportation system, bus identification was identified as designcritical.

3. The control and display conception with the general product development method according to VDI 2221 in combination with a morphological approach has proven to be target-oriented.

4. According to the results to Section 4 the authors suggest four fallback levels for identification (cp. Fig. 5):

1. Level: mobile application with information about the ordered bus (display number, individual routing information).

2. Level: vehicle number for primary identification of the vehicle (in front, rear and on door sides)

3. Level: route information of the bus on the door (individualized to the respective user: clear highlighting of the own stop) for additional security.

4. Level: feedback system to confirm that the user has boarded the right bus (response light upon entering, feedback on the mobile phone as well as route information in the interior).

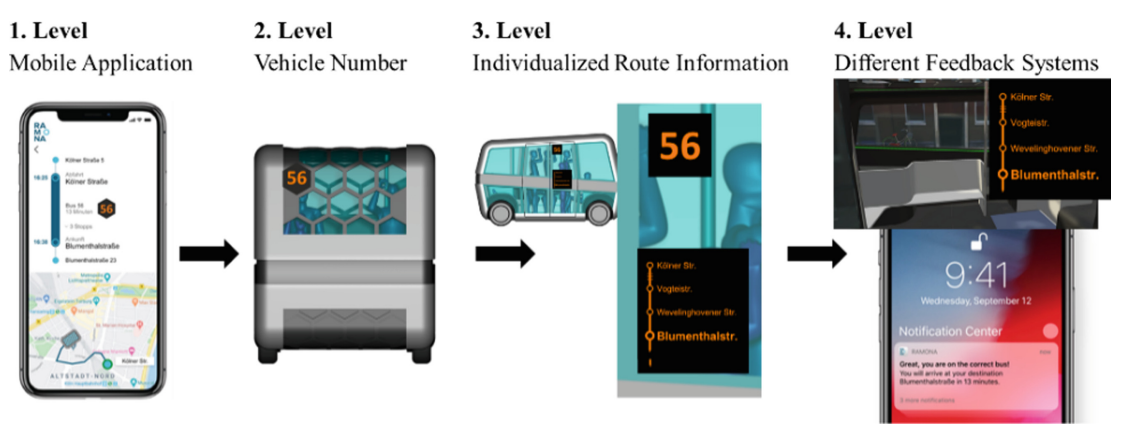

Fig. 5. Proposed fallback levels for correct bus identification.

Acknowledgement. The RAMONA research project was funded by the German Federal Ministry of Transport and Infrastructure. The authors would like to thank on behalf of all project participants for the generous funding. 


\section{References}

1. Springer Medizin. BVG und Charité testen autonome Kleinbusse. Heilberufe 69, 39 (2017). https://doi.org/10.1007/s00058-017-2966-5

2. Brost, M., Klötzke, K., Kopp, G., Deißer, O., Fraedrich, E.-M., Karnahl, K., Sippel, T., Müller, A., Beyer, S.: Development, implementation (pilot) and evaluation of a demand responsive transport system. World Electr. Veh. J. 9, 4 (2018)

3. Mueller, A., Beyer, S., Kopp, G., Deisser, O.: User-centered development of a public transport vehicle operated in a demand-responsive environment. In: Stanton, N. (ed.) Advances in Human Factors of Transportation. AHFE 2019. Advances in Intelligent Systems and Computing, vol. 964, pp. 545 - 555. Springer, Cham (2019)

4. Standard VDI 2221: Design of technical products and systems. Model of product design. Duesseldorf, Germany (2019)

5. Seeger, H.: Basiswissen Transportation Design. Anforderungen, Lösungen, Bewertungen. Wiesbaden, Germany: Springer Fachmedien (2014)

6. Kemper, A., Bubb, I., Kriebel, E., Müller, A.: Identifikation von kritischen Interaktionen des bedarfsgerechten ÖPNV mit autonom betriebenen Fahrzeugen (2020)

7. Salmon, P.M., Young, K.L., Regan, M.A.: Distraction 'on the buses': a novel framework of ergonomics methods for identifying sources and effects of bus driver distraction. Appl. Ergon. 42(4), 602-610 (2011)

8. Feldhusen, J., Grote, K.-H. (eds.): Pahl/Beitz Konstruktionslehre. Methoden und Anwendung erfolgreicher Produktentwicklung. 8. Auflage. Berlin, Heidelberg, Gernmay: Springer, Heidelberg (2013)

9. ISO/IEC. 9241-11. Ergonomic requirements for office work with visual displayterminals (VDTs). The international organization for standardization. 45(9) (1998) 